\title{
Estimación del riesgo en las viviendas de Baja California Sur ante el impacto de ciclones tropicales
}

Recibido: 30/01/2016 · Aceptado: 23/03/2016

\author{
Eleonora Romero Vadillo \\ Universidad Autónoma de Baja California Sur \\ Irma Guadalupe Romero Vadillo
}

Centro Interdisciplinario de Investigaciones y Estudios sobre

Medio Ambiente y Desarrollo, Instituto Politécnico Nacional

\section{Resumen}

En promedio, un ciclón toca tierra cada año en Baja California Sur y aunque predominan como tormenta tropical y huracán categoría 1, los huracanes han impactado a la entidad hasta con categoría 3. Entre los cuantiosos daños que sufre la población los de la vivienda son los más importantes, ya que conllevan un incremento de la marginación. No obstante, el riesgo estimado en función del peligro y la vulnerabilidad no es el mismo para todas las viviendas a lo largo del estado. Para cada municipio el peligro en las viviendas se estimó calculando la probabilidad, por año, de recibir el impacto de un ciclón tropical; por categorías, considerando todas las trayectorias de los huracanes del Pacífico noreste que han golpeado a la entidad de 1966 a 2014. Las viviendas se clasificaron de acuerdo con las características de los materiales de construcción de pisos, techos y muros; su vulnerabilidad se calculó asignando un daño probable a cada tipo de vivienda ante el impacto de un ciclón de determinada intensidad. El índice de vulnerabilidad se configuró con base en la proporción de viviendas en cada nivel de vulnerabilidad para cada uno de los cinco municipios. La Paz y Los Cabos presentan mayor probabilidad de impacto cada año (0.48 y 0.26 , respectivamente, para todas las categorías), mientras que los porcentajes de viviendas más vulnerables se ubican en Loreto, Mulegé y Los Cabos $(4.8,2.9$ y $2.8 \%$, respectivamente). Los resultados muestran que las viviendas de La Paz y Los Cabos son las que ostentan mayor riesgo.

Palabras clave: Vulnerabilidad, riesgo, peligro, viviendas, ciclones tropicales. 


\title{
Risk forecasting for housing in Baja California Sur under tropical cyclone impact
}

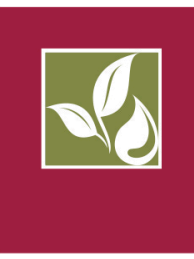

\begin{abstract}
On average a cyclone makes landfall in the state Baja California Sur of every year and although they predominantly do so as tropical storm and category 1 hurricanes, these have impacted as category 3 hurricanes. Among the substantial damages suffered by the population, those on housing are the most important since they generate increased marginalization. However, the estimated risk based on the hazard and vulnerability is not the same for all housing throughout the state. The danger for housing for each of the five municipalities of Baja California Sur was estimated by calculating the probability per year of being hit by a tropical cyclone, by category, considering the paths of hurricanes in the Pacific Northwest between 1966-2014. Housing was classified according to the characteristics of the floor, roof and wall materials and vulnerability was calculated assigning a probable damage to each type of housing under the impact of a cyclone whit a given intensity. The vulnerability index was constructed considering the proportion of houses in each level of vulnerability for each of the 5 municipalities. La Paz and Los Cabos present a higher yearly impact probability ( 0.48 and 0.26 respectively for all categories), while the percentage of higher housing vulnerability are located in the municipalities of Loreto, Mulegé and Los Cabos (4.8\% , 2.9\% and 2.8\% respectively). Results show that housing in the municipalities of La Paz and Los Cabos carry the highest risk. Palabras clave: Vulnerabilidad, Riesgo, Peligro, Viviendas, Ciclones Tropicales
\end{abstract}

KeY worDs: Vulnerability, risk, danger, housing, tropical cyclone. 


\section{Introducción}

Baja California Sur es un estado con alto nivel de riesgo, producto de la combinación de dos factores: las amenazas por ciclones tropicales y la vulnerabilidad de sus edificaciones. Sus condiciones geográficas y climatológicas favorecen el impacto de ciclones tropicales, que traen consigo fuertes vientos e inundaciones derivadas tanto de las lluvias intensas como de la marea de tormenta.

La vivienda es uno de los sectores que resulta con las mayores afectaciones ante la acción de los ciclones tropicales, generándose anualmente cuantiosas pérdidas en el patrimonio de los hogares, lo que conlleva un incremento de la marginación.

Por tanto, es de gran importancia establecer estrategias y programas enfocados a prevenir y reducir los efectos de los fenómenos hidrometeorológicos, y no solo dirigir los recursos a la atención de las emergencias y la reconstrucción.

Para prevenir es indispensable conocer el riesgo, entendido como la pérdida esperada, como resultado de la acción de un fenómeno perturbador sobre un bien expuesto. El riesgo depende, entonces, de dos factores fundamentales: el peligro y la vulnerabilidad (Flores Corona et al., 2004).

Se define el peligro como la probabilidad de que una amenaza se presente con cierta magnitud en un sitio específico, considerándose una amenaza aquellos elementos del ambiente biofísico peligrosos al hombre y que son causados por fuerzas extrañas a él (Chardon y González, 2002, p. 3). Por su parte, la vulnerabilidad se comprendería como el nivel o grado de afectación que puede sufrir un sujeto o elemento cuando es sometido a una amenaza. La vulnerabilidad de una población está en función de la fragilidad de sus elementos (infraestructura, vivienda, actividades productivas, grado de organización, sistemas de alerta, desarrollo político institucional, entre otros). Luego, la vulnerabilidad es la probabilidad de que, dada la intensidad de una amenaza y la fragilidad de los elementos expuestos, ocurran daños en la economía, la vida humana y el ambiente (Chardon y González, 2002, p. 7).

Wisner, Blaikie, Cannon y Davis (2003, p. 11) detallan la vulnerabilidad como las características de una persona o grupo y su situación que influyen en su capacidad para prever, hacer frente, resistir y recuperarse de los efectos de un peligro natural (un evento natural extremo o proceso). Se trata de una combinación de factores que determinan el grado en que la vida, el sustento, 
la propiedad de alguien y otros elementos se ponen en riesgo por un evento o serie en cascada de este tipo de eventos.

De acuerdo con Flores Corona et al. (2004), la estrategia de la prevención implica tres pasos esenciales. Primero, conocer los peligros y amenazas para saber dónde, cuándo y cómo afectan. Segundo, identificar y establecer en el ámbito nacional, estatal, municipal y comunitario, las características y los niveles actuales de riesgo ante esos fenómenos. Por último, diseñar acciones y programas para mitigar y reducir oportunamente estos riesgos a través del reforzamiento y adecuación de la infraestructura, mejorando normas y procurando su aplicación, y finalmente, preparando e informando a la población para que sepa cómo actuar antes, durante y después de una contingencia.

Desde el punto de vista de autores como Wisner et al. (2003: 10), quienes hacen investigación social ligada a los desastres, sin negar la relevancia de los peligros naturales como eventos de disparo, el énfasis se debe poner en las diversas formas en que operan los sistemas sociales para generar desastres volviendo a las personas vulnerables.

Por su parte, Romero y Maskrey (1993: 8) mencionan que la vulnerabilidad en los pueblos ocurre por la ocupación de terrenos que no son aptos para la vivienda, por la construcción de viviendas precarias o hechas con materiales inapropiados para la zona, y por la falta de condiciones económicas para satisfacer sus necesidades, siendo mayor la magnitud de daño real cuando la población carece de recursos con que recuperarse. También señalan que la vulnerabilidad física puede ser de origen, dada la falta de seguridad en las construcciones desde su realización, o progresiva, por el envejecimiento de las mismas.

La estimación de la vulnerabilidad física de las viviendas ante el impacto de ciclones tropicales es una valiosa herramienta que contribuye a proyectar medidas preventivas en el campo de la construcción y rehabilitación de inmuebles vulnerables, siendo cardinal para la protección del patrimonio de las personas, así como para la reducción de la pérdida de vidas humanas mediante la evacuación anticipada de la población que reside en viviendas altamente vulnerables.

El objetivo del presente trabajo es establecer los niveles de riesgo que enfrentan las viviendas de los cinco municipios de Baja California Sur frente al impacto de ciclones tropicales. 


\section{Antecedentes}

El paso de un ciclón tropical puede generar importantes beneficios a las comunidades, principalmente a las zonas áridas, ya que permite la recarga de los mantos acuíferos. Sin embargo, cuando las poblaciones no se encuentran preparadas, puede producir severos daños, como grandes pérdidas económicas y de vidas humanas.

Amén de las afectaciones que pueden causar los fuertes vientos de un ciclón tropical, las intensas precipitaciones, las variaciones de nivel del mar (marea de tormenta) y el oleaje tienen un gran potencial para ocasionar inundaciones en poblaciones costeras, que pueden ser devastadoras para muelles, embarcaciones, viviendas y otras estructuras a lo largo de la costa. El impacto de estos fenómenos en las comunidades depende de muchos factores, que van desde las condiciones geográficas de la región, como la altitud, la presencia de ríos o arroyos, el tipo de suelo y la geomorfología de la zona, hasta la clase y ubicación de las viviendas, además de la intensidad del ciclón (Romero-Vadillo, 2015, p. 70).

La intensidad de un ciclón se mide por la velocidad de sus vientos que pueden llegar hasta los $250 \mathrm{~km} / \mathrm{h}$ en la pared de un huracán de categoría 5, el cual puede presentar ráfagas que exceden los $360 \mathrm{~km} / \mathrm{h}$. El poder destructivo del viento aumenta con el cuadrado de su velocidad (oEA, 1993, cap. 12). La devastación es causada tanto por impacto directo del viento como por el material que acarrea el aire. Las edificaciones construidas por el hombre son altamente vulnerables. Los daños ocasionados por los vientos pueden ir desde algunos menores durante una tormenta tropical, hasta graves en edificios y casas-habitación, por ejemplo la pérdida de la techumbre y algunas paredes, así como la destrucción total de edificaciones ligeras y temporales durante un huracán de categoría 5 (Flores Corona et al., 2004). Cuando la intensidad del viento es de tal magnitud que la presión con la que empuja supera la resistencia de los materiales (concreto, acero, mampostería, madera), los elementos estructurales (muros de carga, columnas, vigas o trabes, losas, escaleras y cimientos) se dañan. Las viviendas también pueden sufrir afectaciones si hay errores constructivos o de diseño, o la calidad de los materiales no es la adecuada, asimismo, si la cimentación no fue apropiadamente diseñada para las características del terreno de apoyo, y para soportar las fuerzas que le transmite la estructura. En general, un muro pesado de mampostería o adobe no es afectado por el viento, 
pero sí lo es un techo ligero, ventanales, e incluso bardas o muros aislados, deficientemente reforzados. No obstante, el daño ocasionado depende también de qué tan expuesta está la zona (entre árboles, entre edificios altos, en campo libre, en la costa, etc.) (Cenapred, s. f.).

Otra amenaza que se manifiesta al paso de un ciclón tropical la constituyen las intensas precipitaciones, las cuales son extremadamente variables y difíciles de predecir (Flores Corona et al., 2004); pueden resultar muy intensas y durar varios días, o ligeras y de corta duración. La precipitación no se encuentra directamente correlacionada con la intensidad de los vientos, esta depende de la topografía local y la humedad, así como de la conformación del campo nuboso y la velocidad de avance del huracán (Romero-Vadillo, 2015, p. 25). Las inundaciones generadas por la abundante precipitación pueden afectar desde los cimientos y paredes, hasta el techo de una vivienda. Los sistemas eléctricos y los electrodomésticos presentan graves daños durante una inundación.

Como peligros secundarios, asociados a las abundantes precipitaciones, se hallan los deslizamientos de tierra que ocurren cuando las áreas con pendientes desde medianas hasta muy pronunciadas se sobresaturan y fallan, los cuales son capaces de derrumbar viviendas localizadas en las zonas altas o laderas o dañar aquellos asentamientos ubicados en las faldas del cerro que sufre un deslave.

Una amenaza más que se enfrenta ante un ciclón tropical es la marea de tormenta, que consiste en un aumento anormal del nivel del mar, con dimensiones que van desde $0.5 \mathrm{~m}$ de altura para ciclones de poca intensidad, pero que pueden alcanzar más de $7 \mathrm{~m}$ durante huracanes intensos. Las mareas de tormenta son generadas por la combinación de dos procesos: cambios en el nivel medio del mar causados por modificaciones en la presión atmosférica y el movimiento del agua que es impulsada hacia tierra por la fuerza del viento. Se estima una elevación del nivel del mar de $1 \mathrm{~m}$ por cada 100 mbar que disminuya la presión barométrica. No obstante, la magnitud de la marea en un determinado lugar también está en función del radio de los vientos máximos del huracán, la velocidad de avance del sistema, así como de la morfometría del sitio y la batimetría frente a la costa. Al elevarse el nivel del mar, el agua entra a la zona costera, afectando las áreas bajas varios kilómetros tierra adentro y trasladando la rompiente del oleaje también tierra adentro, por lo cual el efecto de la marea 
de tormenta, aunado al oleaje producido por el ciclón, es devastador para las viviendas y toda la infraestructura situada cerca de la línea de costa. Cuando el efecto de la marea de tormenta se combina con marea alta, los resultados son aún más catastróficos (Romero-Vadillo, 2015, p. 62). Sumado a ello, el aumento del nivel del mar bloquea la salida de las aguas de los ríos hacia el mar y, en consecuencia, el agua no tiene por donde escapar, potenciando las inundaciones (OEA, 1993, cap. 12).

De acuerdo con los registros históricos de la Administración Nacional Atmosférica y Oceánica (National Oceanic and Atmospheric Administration, NOAA), de 1966 a 2015 se consignaron 125 ciclones tropicales en el Pacífico noreste con impacto directo en México, de los cuales 62 fueron huracanes, 11 de ellos calificados como intensos (categorías 3, 4 y 5 en la escala Saffir-Simpson). Conforme a este registro histórico, las entidades de las costas del Pacífico mexicano con mayor riesgo ciclónico son Baja California Sur con 44, Sinaloa con 21 y Jalisco con 15 (considerando el estado donde el ciclón toca tierra por primera vez) (figura 1). Estos impactos han tenido consecuencias severas en términos de pérdida de vidas, daños físicos en los inmuebles y los enseres, así como afectaciones en la estructura carretera y los servicios.

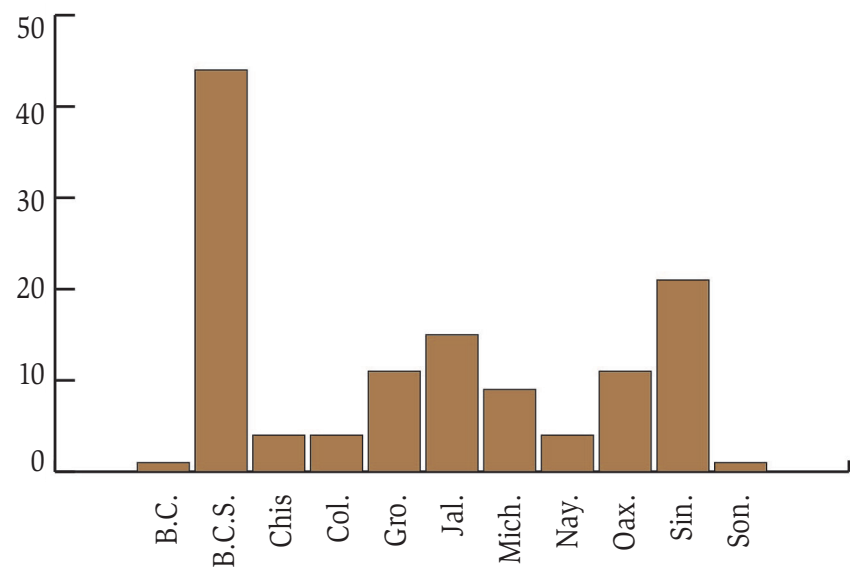

Fuente: Elaboración propia con información del National Hurricane Center de la NOAA.

FiguRa 1. Número de ciclones tropicales del Pacífico noreste que han impactado como primer punto cada entidad entre 1966 y 2015 
Baja California Sur se ubica al noroeste de México, ocupando la mitad sur de la península de Baja California. Limita al norte con el estado de Baja California, al este con el Mar de Cortés y al sur y oeste con el océano Pacífico. Tiene una superficie de $73475 \mathrm{~km}^{2}$ y está conformado por cinco municipios: Los Cabos, La Paz, Comondú, Loreto y Mulegé (figura 2). Es una región árida que posee el promedio de lluvia más bajo de todo el país. Cuenta con un gran número de amplios arroyos que permanecen secos la mayor parte del tiempo, en ocasiones durante varios años, lo que origina el asentamiento de viviendas en sus márgenes o incluso en el cauce mismo.

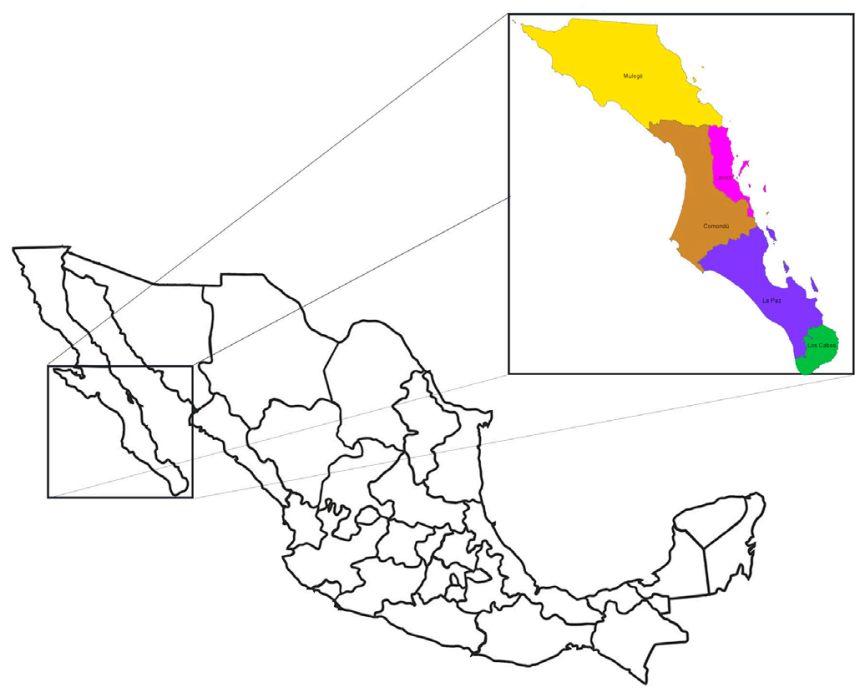

Fuente: Elaboración propia.

Figura 2. Ubicación y división política de Baja California Sur

En promedio, un ciclón impacta Baja California Sur cada año, produciendo intensas precipitaciones que conllevan la corrida de los arroyos, inundando tales viviendas. Aunado a esto, los fuertes vientos y la marea de tormenta causan graves daños a las viviendas. Asimismo existen algunas asentadas en zonas de deslaves, las cuales también son altamente propensas a sufrir daños. 
El impacto de los ciclones tropicales ha sido importante en los últimos años, sobresaliendo el generado por los siguientes huracanes:

- Olivia (1967), el cual impactó con categoría 3 de huracán las poblaciones de Santa Rosalía, Loreto y Mulegé. No existen cifras de los daños materiales ocasionados por este ciclón, sin embargo, se sabe que 61 personas perdieron la vida (Romero-Vadillo, 2015, p. 129).

- Liza (1976) es uno de los más intensos de los que se han dejado sentir en Baja California Sur, aun cuando el ojo del huracán no tocó tierra en la entidad, pasando aproximadamente a $30 \mathrm{~km}$ de ella. No obstante, debido a su cercanía e intensidad a su paso frente a las costas de Baja California Sur (vientos de $222 \mathrm{~km} / \mathrm{h}$ ), el impacto fue grande. Las precipitaciones en los municipios de La Paz y Los Cabos fueron abundantes, registrándose hasta $559 \mathrm{~mm}$ en 24 horas. Las intensas lluvias provocaron que una presa, ubicada en el arroyo El Cajoncito, en la ciudad de La Paz, se reventara arrasando con una colonia entera, lo que ocasionó la pérdida de más de 600 vidas (Romero-Vadillo, 2015, p. 130).

- Kiko (1989) arribó a Baja California Sur con categoría 3. La precipitación alcanzó $302 \mathrm{~mm}$ en 24 horas en Santiago, municipio de Los Cabos, y causó graves daños en todo el extremo sur de la península, destruyendo gran número de viviendas, embarcaciones, muelles y vías de comunicación. El 13 de septiembre tocó tierra en Todos Santos, Baja California Sur, con categoría 1, presentando vientos de $140 \mathrm{~km} / \mathrm{h}$. La precipitación llegó a $200 \mathrm{~mm}$ en 24 horas en el municipio de Los Cabos y a $130 \mathrm{~mm}$ en La Paz (Romero-Vadillo, 2015, p. 132).

- Paúl (1982) impactó la entidad con categoría 2; el saldo en San José del Cabo fue de 9000 personas sin hogar, mientras que en La Paz al menos 85 viviendas sufrieron afectaciones.

- Fausto (1996) llegó al sur de la península con categoría 1 ocasionando precipitaciones máximas de $200 \mathrm{~mm}$ en 24 horas. Los arroyos corrieron interrumpiendo las carreteras en varios tramos. Los daños en todo el estado fueron considerables y una persona murió (Romero-Vadillo, 2015, p. 132). 
- Juliette (2001) permaneció prácticamente estacionado durante tres días frente a las costas del sur de la península; la precipitación registrada alcanzó una cifra histórica para el estado. En Santiago, municipio de Los Cabos, la precipitación acumulada en tres días fue de $915 \mathrm{~mm}$, mientras que en la estación El Rosario, en el municipio de La Paz, llegó a $650 \mathrm{~mm}$. A consecuencia de la abundante lluvia, los arroyos se desbordaron, provocando grandes inundaciones, las cuales tuvieron como resultado dos muertos, 11000 damnificados, 4000 viviendas, 39 planteles educativos y 72 espacios deportivos dañados o destruidos; asimismo, más de $1300 \mathrm{~km}$ de la red de carreteras y caminos rurales resultaron severamente dañados. Todos los servicios quedaron suspendidos (Romero-Vadillo, 2015, p. 134).

- En 2003, dos huracanes golpearon Baja California Sur: Ignacio, el 24 de agosto, con categoría 1, y Marty, el 22 de septiembre, con categoría 2; ambos produjeron daños considerables en la vivienda y la infraestructura. Marty dejó a su paso 12 muertos, ocho heridos y 4000 viviendas destruidas (Romero-Vadillo, 2015, pp. 136-140).

- John (2006) entró a Baja California Sur con categoría 2, azotando todas las comunidades del sur del estado y, ya como tormenta tropical, atravesó los municipios de Comondú, Loreto y Mulegé. El paso de John por el sur de la península generó cuantiosos daños, un gran número de damnificados y seis muertos. En el municipio de La Paz, las abundantes precipitaciones (por encima de los $250 \mathrm{~mm}$ ) provocaron el desbordamiento del arroyo Las Bramonas, con la consecuente inundación de varias casas. La comunidad más afectada fue Mulegé, donde se presentaron lluvias superiores a los $200 \mathrm{~mm}$ en 24 horas, esto es, el doble de la media anual, causando el desbordamiento del río del mismo nombre, el cual inundó cerca de 450 casas, una escuela secundaria y una preparatoria, cubriéndolas casi por completo, perdiendo sus habitantes muchos de sus bienes materiales. Asimismo resultaron afectadas varias casas rodantes que se llevó el río. Santa Rosalía también sufrió grandes daños debido a la marea de tormenta, sepultando autos e incluso arrastrándolos mar adentro (Romero-Vadillo, 2015, pp. 142-145). 
- Henriette (2007) impactó el estado con categoría 1, generando importantes afectaciones en los servicios, 2200 viviendas resultaron dañadas en el municipio de Los Cabos y una persona perdió la vida. Tres de los municipios fueron declarados en desastre (Cenapred, 2009, pp. 249-251).

- Norbert (2008) ingresó a tierras sudcalifornianas con categoría 2, provocando cuantiosas inundaciones, la suspensión de los servicios, así como la interrupción en las vías de comunicación principalmente en los municipios de La Paz, Comondú y Loreto. En estos dos últimos, 4000 personas perdieron sus viviendas. En las islas de Margarita y Magdalena, $90 \%$ de las viviendas de los pescadores fueron destruidas (Rodríguez, 2008).

- Jimena (2009) tocó tierra con categoría 2, y aunque lo hizo en el municipio de Comondú, el radio de vientos y precipitación afectó a todo el estado. A su paso por Comondú, Jimena se llevó viviendas completas en localidades pesqueras, arrasó con el centro comercial, calles e infraestructura urbana de Ciudad Constitución, que fue golpeada por fuertes vientos durante siete horas. Un total de 2700 viviendas resultaron dañadas en todo el estado. Las afectaciones en los servicios y la infraestructura carretera fueron cuantiosas (Romero-Vadillo y RomeroVadillo, 2014, p. 8).

- Odile (2014) azotó la entidad con categoría 3, siendo, después de Liza, el huracán que más daños ha generado en Baja California Sur. Odile provocó seis muertes, 30000 personas fueron llevadas a uno de los 164 albergues instalados y 26000 turistas tuvieron que ser evacuados mediante un puente aéreo, días después del impacto (CNNMéxico, 2014); 25860 viviendas fueron afectadas, de las cuales 1877 sufrieron daño total. Las pérdidas económicas ascendieron a más de 7800 millones de pesos, sin considerar los daños causados en la infraestructura de la Comisión Federal de Electricidad. En cuanto a servicios básicos, $92 \%$ de la población no tuvo acceso a energía eléctrica por más de una semana, mismo tiempo que demoró el servicio de telecomunicaciones, mientras que el de agua potable tardó cerca de dos semanas en ser reinstalado (Velasco, 2014). 
Los cuantiosos daños que han sufrido las viviendas en Baja California Sur a consecuencia del impacto de los ciclones tropicales hace necesaria la estimación del riesgo que estas presentan, a fin de crear una cultura de la prevención en la comunidad. No obstante, hay que considerar que el riesgo es una variable muy compleja y continuamente cambiante, que se modifica en función de la variabilidad de las amenazas y de la condición, también dinámica, de la vulnerabilidad y el grado de exposición, derivados de factores socioeconómicos y del desarrollo, como los elevados índices de construcciones informales, marginación, pobreza y escaso ordenamiento urbano y territorial, entre otros; por lo cual este debe ser estimado de acuerdo con las circunstancias y condiciones específicas del lugar (Flores Corona et al., 2004).

\section{Metodología}

El peligro, para cada categoría, se estimó calculando la probabilidad de recibir el impacto de un ciclón de esa categoría, para lo cual se consideraron todas las trayectorias de los huracanes del Pacífico noreste que han azotado a la entidad de 1966 a 2015.

La probabilidad se calculó mediante la distribución de Poisson. El valor de $\lambda$ se estimó contabilizando el número de ciclones de cada categoría que han pasado por el municipio en ese periodo, dividido entre el número de años que abarca (50), siendo este el estimador de máxima verosimilitud, esto es:

$$
\lambda_{\mathrm{c}}=\frac{\mathrm{n}_{\mathrm{c}}}{50}
$$

Donde $\mathrm{n}_{\mathrm{c}}$ es el número de ciclones de categoría $c$ que han impactado en el municipio.

Luego, la probabilidad de que al menos un ciclón de esa categoría impacte el municipio en un año determinado está dada por:

$$
\mathrm{P}_{\mathrm{C}}=1-\mathrm{e}^{-\lambda c} \quad \mathrm{c}=1,2, \ldots 7
$$


Donde:

$c$ es la categoría $(1=\mathrm{DT}, 2=\mathrm{TT}, 3=\mathrm{C} 1,4=\mathrm{C} 2,5=\mathrm{C} 3,6=\mathrm{C} 4,7=\mathrm{C} 5)$

$\mathrm{P}_{\mathrm{C}}$ es la probabilidad de que al menos un ciclón de categoría $c$ impacte en el municipio.

El periodo de retorno para cada categoría $\operatorname{Ret}_{C}$ en cada uno de los municipios se estimó como el inverso de $\lambda$, esto es:

$$
\operatorname{Ret}_{c}=\frac{1}{\lambda_{c}}
$$

La función de vulnerabilidad se define como la relación matemática que asocia las consecuencias de un fenómeno sobre una construcción, una obra de ingeniería, o un conjunto de bienes o sistemas expuestos, con la intensidad del mismo (Mendoza-Estrada, Vélez-Morales, Fuentes-Mariles, De Luna-Cruz y Pelaez-Pavón, 2014, p. 1).

La vulnerabilidad, en este trabajo, se expresa como un valor entre cero y uno, que equivale al daño probable ante un ciclón de cierta categoría. Cero implica que el daño probable en las viviendas ante un evento de cierta intensidad es nulo, y uno, que el daño que puede sufrir la vivienda es total.

De acuerdo con Flores Corona et al. (2004), los daños ocasionados por un ciclón tropical, según su categoría, se muestran en el cuadro 1, siendo los daños acumulativos en las subsecuentes categorías.

Para el cálculo de la vulnerabilidad física de las viviendas se empleó una clasificación en función de las características de estas, que las hacen susceptibles al daño por viento, lluvia e inundación, a partir de la información proporcionada por el Instituto Nacional de Estadística y Geografía (INEGI) en el cuestionario ampliado de la muestra censal 2010, el cual contiene todas las preguntas del cuestionario básico del Censo de Población y Vivienda y preguntas adicionales relativas a las características de las viviendas y sus habitantes. La información al respecto con que cuenta el INEGi clasifica a la vivienda por el material usado en paredes, techos y pisos. Cada uno de estos elementos se agrupó en tres categorías (alta, media y baja), de acuerdo con la resistencia de los materiales:

a) Paredes:

- Alta: tabique, ladrillo, block, piedra, cantera o cemento o concreto.

- Media: madera o adobe. 
CuAdro 1. Daño esperado en las viviendas de acuerdo con la intensidad de impacto de un ciclón tropical

\begin{tabular}{|c|c|c|c|}
\hline CAtegoría & $\begin{array}{l}\text { Velocidad del } \\
\text { viento }(\mathrm{km} / \mathrm{h})\end{array}$ & $\begin{array}{l}\text { Marea de } \\
\text { tormenta (m) }\end{array}$ & Daños en la vivienda \\
\hline $\begin{array}{l}\text { Depresión } \\
\text { tropical }\end{array}$ & $<63$ & - & $\begin{array}{l}\text { Puede causar daño en pisos, paredes } \\
\text { y enseres en viviendas precarias o } \\
\text { establecidas en zonas de inundación. }\end{array}$ \\
\hline $\begin{array}{l}\text { Tormenta } \\
\text { tropical }\end{array}$ & $63-116$ & $<1$ & $\begin{array}{l}\text { Daños en los techos de algunas } \\
\text { viviendas con techo de cartón negro o } \\
\text { lámina, que no estén en buen estado. }\end{array}$ \\
\hline Huracán 1 & $119-153$ & $1.5-1.9$ & $\begin{array}{l}\text { No se presenta daño real en estructuras } \\
\text { de edificios. Esquinas de techos } \\
\text { removidas, casas móviles arrastradas. }\end{array}$ \\
\hline Huracán 2 & $154-177$ & $2.0-2.5$ & $\begin{array}{l}\text { Daño en materiales de techos, } \\
\text { puertas y ventanas de edificios. Daño } \\
\text { considerable en casas móviles. }\end{array}$ \\
\hline Huracán 3 & $178-209$ & $2.6-4.4$ & $\begin{array}{l}\text { Daño estructural en pequeñas casas } \\
\text { habitación. Destrucción total de casas } \\
\text { móviles. }\end{array}$ \\
\hline Huracán 4 & $210-249$ & $4.5-5.0$ & $\begin{array}{l}\text { Fallas y daño extenso en muros } \\
\text { de colindancia, con algunas fallas } \\
\text { completas en techos de algunas casas } \\
\text { habitación. }\end{array}$ \\
\hline Huracán 5 & $>250$ & $>5.0$ & $\begin{array}{l}\text { Daño y falla completa en techumbres, } \\
\text { edificios y casas habitación. Falla } \\
\text { completa de edificaciones ligeras y } \\
\text { temporales. }\end{array}$ \\
\hline
\end{tabular}

Fuente: Elaboración propia con información de (Flores-Corona et al., 2006)

- Baja: material de desecho, lámina de cartón, lámina de asbesto o metálica, carrizo, bambú o palma, embarro o bajareque.

b) Techos:

- Alta: losa de concreto o viguetas con bovedilla o terrado con viguería.

- Media: lámina metálica, lámina de asbesto, madera o tejamanil o teja.

- Baja: material de desecho, lámina de cartón o palma o paja.

c) Pisos:

- Alta: madera, mosaico u otro recubrimiento.

- Media: cemento o firme.

- Baja: tierra. 
Con la combinación de estas categorías se establecieron 27 tipos de vivienda. Con base en los daños asociados a cada categoría y la clasificación de las viviendas según sus características, se determinó el daño probable para cada tipo de vivienda y cada categoría de impacto.

A partir de los datos extraídos del Censo de Población y Vivienda 2010 (INEGI, 2010) se obtuvo la proporción de viviendas en cada nivel de clasificación por municipio. El índice de vulnerabilidad física para cada categoría (IV ) por municipio se sacó sumando el producto del porcentaje de daño de cada categoría por el porcentaje de viviendas en cada nivel, por municipio.

$$
I V c=\sum_{\mathrm{r}=1}^{5} D_{c r} q_{r}
$$

Donde:

$D_{C r}$ es la proporción de daño probable del $r$-ésimo tipo de vivienda ante un impacto de categoría $c$.

$q_{r}$ es la proporción de viviendas del r-ésimo tipo.

El riesgo se define como el valor esperado de pérdidas de cierto elemento, considerando la probabilidad de ocurrencia de determinada amenaza. En el presente trabajo se entiende como el daño esperado en las viviendas de cada municipio de Baja California Sur en una temporada de ciclones.

El riesgo, estimado en función del peligro y la vulnerabilidad, se calculó para cada municipio de la siguiente forma:

$$
R=\sum_{\mathrm{C}=1}^{7} P_{C} I V_{C}
$$

\section{Resultados}

Baja California Sur es directamente impactada en promedio por un ciclón tropical cada año, y aunque predomina el impacto como tormenta tropical y huracán categoría 1, estos han golpeado a la entidad hasta con categoría 3 de huracán. De 1966 a 2015, 45 ciclones han tocado tierra en el estado (figura 3). 


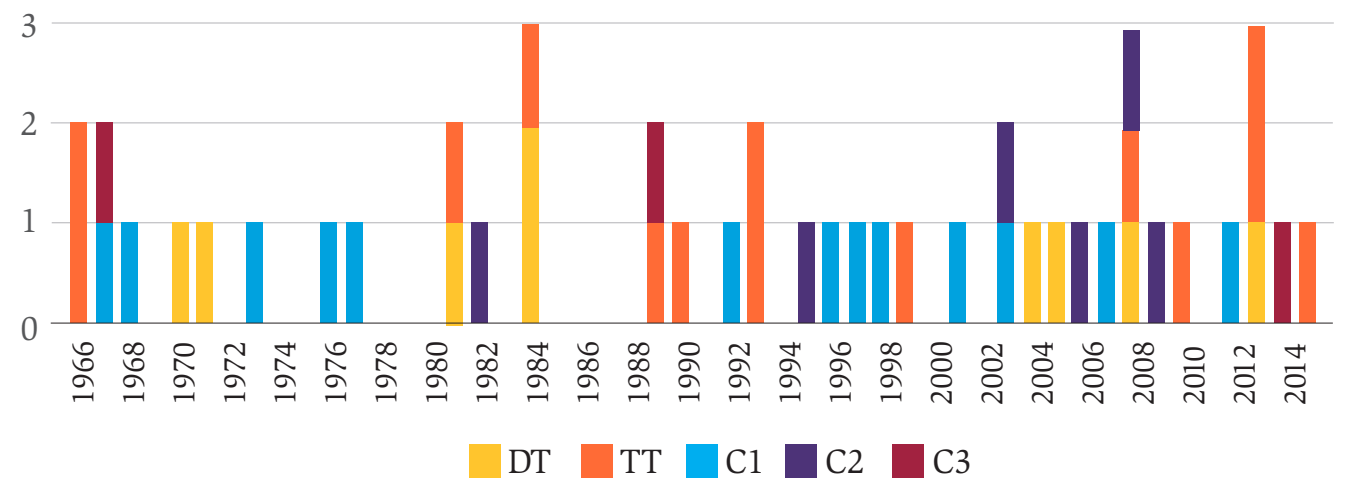

Fuente: Elaboración propia con datos del National Hurricane Center de la NOAA.

Figura 3. Número de ciclones que han tocado tierra en Baja California Sur de acuerdo con la categoría de impacto

Sin embargo, el impacto no es el mismo para cada uno de los cinco municipios. La figura 4 muestra las trayectorias de todos los ciclones que han arribado a Baja California Sur entre 1996 y 2015, indicando mediante colores su categoría.

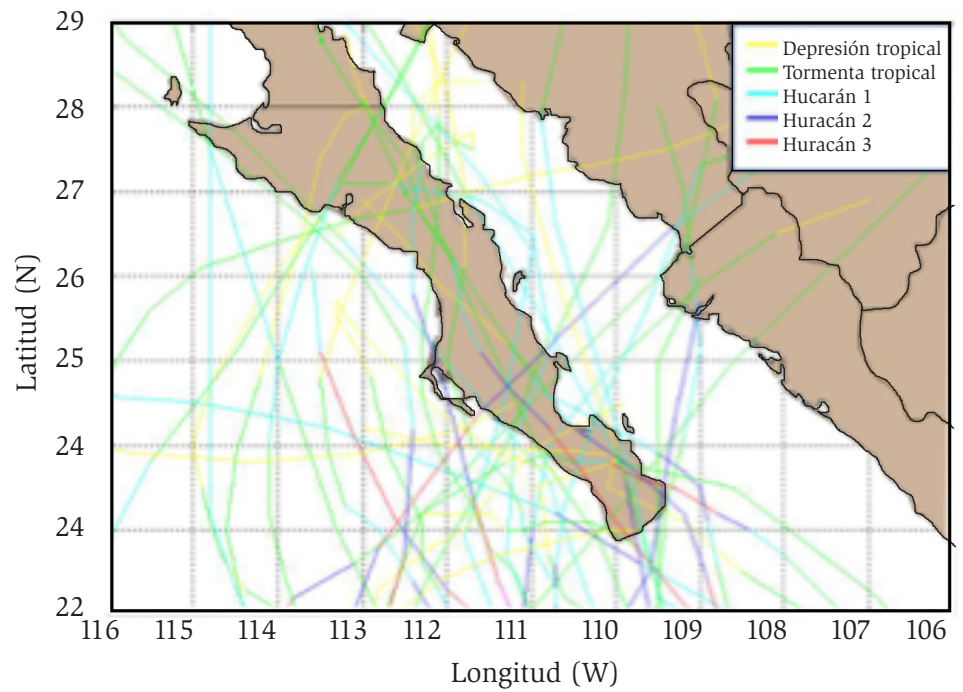

Fuente: Elaboración propia con datos del National Hurricane Center de la noaa.

Figura 4. Trayectoria de los ciclones que han impactado Baja California Sur (1966-2015) 
En el cuadro 2 se presenta el número de ciclones que han tocado tierra en cada municipio, de acuerdo con su categoría de impacto, así como su frecuencia relativa $(\lambda)$, el periodo de retorno y la probabilidad de que al menos un ciclón de esa intensidad impacte el municipio en un año. No se consideraron las categorías 4 y 5 de huracán, ya que el ojo de ningún ciclón ha tocado tierra en Baja California Sur con tales intensidades.

CUADRo 2. Número de huracanes que han tocado tierra en Baja California Sur entre 1966 y 2015, frecuencia relativa, periodo de retorno y probabilidad estimada (P) de que al menos n ciclón toque tierra por categoría de impacto

\begin{tabular}{|c|c|c|c|c|c|}
\hline Municipio & Categoría & Impactos & $\begin{array}{l}\text { Frecuencia } \\
\text { relativa }(\lambda)\end{array}$ & $\begin{array}{l}\text { Periodo de } \\
\text { retorno }\end{array}$ & P \\
\hline \multirow[t]{5}{*}{ Los Cabos } & DT & 1 & 0.02 & 50 & 0.02 \\
\hline & $\mathrm{TT}$ & 5 & 0.1 & 10 & 0.1 \\
\hline & $\mathrm{C} 1$ & 1 & 0.02 & 50 & 0.02 \\
\hline & $\mathrm{C} 2$ & 4 & 0.08 & 12.5 & 0.08 \\
\hline & $\mathrm{C} 3$ & 2 & 0.04 & 25 & 0.04 \\
\hline \multirow[t]{5}{*}{ La Paz } & DT & 6 & 0.12 & 8.3 & 0.11 \\
\hline & $\mathrm{TT}$ & 6 & 0.12 & 8.3 & 0.11 \\
\hline & $\mathrm{C} 1$ & 9 & 0.18 & 5.6 & 0.16 \\
\hline & $\mathrm{C} 2$ & 2 & 0.04 & 25 & 0.04 \\
\hline & C3 & 1 & 0.02 & 50 & 0.02 \\
\hline \multirow[t]{5}{*}{ Loreto } & DT & 2 & 0.04 & 25 & 0.04 \\
\hline & $\mathrm{TT}$ & 2 & 0.04 & 25 & 0.04 \\
\hline & $\mathrm{C} 1$ & 2 & 0.04 & 25 & 0.04 \\
\hline & $\mathrm{C} 2$ & 0 & 0 & $>50$ & 0 \\
\hline & C3 & 0 & 0 & $>50$ & 0 \\
\hline \multirow[t]{7}{*}{ Comondú } & DT & 0 & 0 & $>50$ & 0 \\
\hline & $\mathrm{TT}$ & 9 & 0.18 & 5.6 & 0.16 \\
\hline & $\mathrm{C} 1$ & 2 & 0.04 & 25 & 0.04 \\
\hline & $\mathrm{C} 2$ & 2 & 0.04 & 25 & 0.04 \\
\hline & C3 & 0 & 0 & $>50$ & 0 \\
\hline & DT & 7 & 0.14 & 7.1 & 0.12 \\
\hline & $\mathrm{TT}$ & 6 & 0.12 & 8.3 & 0.11 \\
\hline \multirow[t]{3}{*}{ Mulegé } & $\mathrm{C} 1$ & 5 & 0.1 & 10 & 0.09 \\
\hline & $\mathrm{C} 2$ & 0 & 0 & $>50$ & 0 \\
\hline & C3 & 0.02 & 0.02 & 50 & 0.02 \\
\hline
\end{tabular}


Con base en los daños asociados a cada categoría, descritos anteriormente, y la clasificación de las viviendas en función de sus características, se determinó el daño probable para cada tipo de vivienda y cada categoría de impacto. Los resultados se exhiben en el cuadro 3 .

CuAdRo 3. Proporción de daño probable de acuerdo al tipo de vivienda y categoría de impacto

\begin{tabular}{|c|c|c|c|c|}
\hline \multicolumn{5}{|c|}{ Depresión tropical } \\
\hline \multirow{2}{*}{$\begin{array}{c}\text { Tipo de } \\
\text { pared }\end{array}$} & \multirow{2}{*}{$\begin{array}{l}\text { Tipo de } \\
\text { piso }\end{array}$} & \multicolumn{3}{|c|}{ Tipo de techo } \\
\hline & & Alta & Media & Baja \\
\hline \multirow{3}{*}{ Alta } & Alta & 0.00 & 0.05 & 0.10 \\
\hline & Media & 0.00 & 0.05 & 0.12 \\
\hline & Baja & 0.05 & 0.10 & 0.18 \\
\hline \multirow{3}{*}{ Media } & Alta & 0.05 & 0.08 & 0.12 \\
\hline & Media & 0.08 & 0.10 & 0.15 \\
\hline & Baja & 0.10 & 0.15 & 0.20 \\
\hline \multirow{3}{*}{ Baja } & Alta & 0.15 & 0.18 & 0.20 \\
\hline & Media & 0.18 & 0.20 & 0.25 \\
\hline & Baja & 0.20 & 0.25 & 0.30 \\
\hline
\end{tabular}

\begin{tabular}{|c|c|c|c|c|}
\hline \multicolumn{5}{|c|}{ Tormenta tropical } \\
\hline \multirow{2}{*}{$\begin{array}{c}\text { Tipo de } \\
\text { pared }\end{array}$} & \multirow{2}{*}{$\begin{array}{l}\text { Tipo de } \\
\text { piso }\end{array}$} & \multicolumn{3}{|c|}{ Tipo de techo } \\
\hline & & Alta & Media & Baja \\
\hline \multirow[t]{3}{*}{ Alta } & Alta & 0.03 & 0.07 & 0.14 \\
\hline & Media & 0.04 & 0.07 & 0.17 \\
\hline & Baja & 0.06 & 0.13 & 0.25 \\
\hline \multirow[t]{3}{*}{ Media } & Alta & 0.06 & 0.10 & 0.17 \\
\hline & Media & 0.10 & 0.13 & 0.21 \\
\hline & Baja & 0.12 & 0.20 & 0.28 \\
\hline \multirow[t]{3}{*}{ Baja } & Alta & 0.18 & 0.23 & 0.28 \\
\hline & Media & 0.22 & 0.26 & 0.35 \\
\hline & Baja & 0.24 & 0.33 & 0.42 \\
\hline
\end{tabular}

\begin{tabular}{cllll}
\multicolumn{6}{c}{$\begin{array}{c}\text { Tipo de } \\
\text { pared }\end{array}$} & $\begin{array}{c}\text { Tipo de } \\
\text { piso }\end{array}$ & Alta & Media & Baja \\
\hline Alta & Alta & 0.04 & 0.08 & 0.20 \\
& Media & 0.05 & 0.08 & 0.24 \\
& Baja & 0.07 & 0.17 & 0.35 \\
Media & Alta & 0.07 & 0.14 & 0.24 \\
& Media & 0.12 & 0.17 & 0.29 \\
& Baja & 0.14 & 0.25 & 0.39 \\
& Alta & 0.22 & 0.30 & 0.39 \\
& Media & 0.26 & 0.34 & 0.49 \\
& Baja & 0.29 & 0.42 & 0.59 \\
\hline
\end{tabular}

\begin{tabular}{|c|c|c|c|c|}
\hline \multicolumn{5}{|c|}{ Categoría 2} \\
\hline \multirow{2}{*}{$\begin{array}{c}\text { Tipo de } \\
\text { pared }\end{array}$} & \multirow{2}{*}{$\begin{array}{l}\text { Tipo de } \\
\text { piso }\end{array}$} & \multicolumn{3}{|c|}{ Tipo de techo } \\
\hline & & Alta & Media & Baja \\
\hline \multirow[t]{3}{*}{ Alta } & Alta & 0.04 & 0.11 & 0.27 \\
\hline & Media & 0.06 & 0.11 & 0.33 \\
\hline & Baja & 0.09 & 0.22 & 0.49 \\
\hline \multirow[t]{3}{*}{ Media } & Alta & 0.09 & 0.18 & 0.33 \\
\hline & Media & 0.14 & 0.22 & 0.41 \\
\hline & Baja & 0.17 & 0.33 & 0.55 \\
\hline \multirow[t]{3}{*}{ Baja } & Alta & 0.26 & 0.40 & 0.55 \\
\hline & Media & 0.31 & 0.44 & 0.69 \\
\hline & Ваја & 0.35 & 0.55 & 0.82 \\
\hline
\end{tabular}


CUADRo 3. Proporción de daño probable de acuerdo al tipo de vivienda y categoría de impacto

(finaliza)

\begin{tabular}{clccc}
\multicolumn{5}{c}{ Categoría 3 } \\
$\begin{array}{c}\text { Tipo de } \\
\text { pared }\end{array}$ & $\begin{array}{c}\text { Tipo de } \\
\text { piso }\end{array}$ & \multicolumn{2}{c}{ Tipo de techo } \\
& & Alta & Media & Baja \\
\hline \multirow{4}{*}{ Alta } & Alta & 0.05 & 0.14 & 0.38 \\
& Media & 0.07 & 0.14 & 0.46 \\
& Baja & 0.10 & 0.29 & 0.69 \\
\multirow{3}{*}{ Media } & Alta & 0.10 & 0.23 & 0.46 \\
& Media & 0.17 & 0.29 & 0.58 \\
& Baja & 0.21 & 0.43 & 0.77 \\
Baja & Alta & 0.31 & 0.51 & 0.77 \\
& Media & 0.37 & 0.57 & 0.96 \\
& Baja & 0.41 & 0.71 & 1.00 \\
\hline
\end{tabular}

De acuerdo con el Censo de Población y Vivienda 2010 (INEGI, 2010), Baja California Sur cuenta con 186628 viviendas distribuidas en los cinco municipios de la siguiente manera: Los Cabos, 74261 habitantes; La Paz, 71 825; Comondú, 19 171; Loreto, 5014 y Mulegé, 16 357. Para cada municipio se obtuvo el porcentaje de viviendas de cada tipo. Los resultados aparecen en el cuadro 4.

Cuadro 4. Porcentaje de viviendas de cada tipo en cada uno de los municipios de Baja California Sur

\begin{tabular}{clccc}
\multicolumn{6}{c}{$\begin{array}{c}\text { Tipo de } \\
\text { pared }\end{array}$} & $\begin{array}{c}\text { Tipo de } \\
\text { piso }\end{array}$ & \multicolumn{2}{c}{ Tipo de techo } \\
& & Alta & Media & Baja \\
\hline \multirow{4}{*}{ Alta } & Alta & 0.533 & 0.003 & 0.002 \\
& Media & 0.301 & 0.035 & 0.011 \\
& Baja & 0.011 & 0.005 & 0.005 \\
& Alta & 0.000 & 0.000 & 0.000 \\
Media & Media & 0.000 & 0.019 & 0.013 \\
& Baja & 0.000 & 0.006 & 0.016 \\
& Alta & 0.001 & 0.000 & 0.000 \\
Baja & Media & 0.002 & 0.001 & 0.007 \\
& Baja & 0.000 & 0.001 & 0.028 \\
\hline
\end{tabular}

\begin{tabular}{cllll}
\multicolumn{1}{c}{$\begin{array}{c}\text { Tipo de } \\
\text { pared }\end{array}$} & $\begin{array}{c}\text { Tipo de } \\
\text { piso }\end{array}$ & \multicolumn{3}{c}{ Tipo de techo } \\
& & Alta & Media & Baja \\
\hline \multirow{4}{*}{ Alta } & Alta & 0.419 & 0.006 & 0.001 \\
& Media & 0.417 & 0.082 & 0.008 \\
& Baja & 0.005 & 0.004 & 0.001 \\
\multirow{4}{*}{ Media } & Alta & 0.000 & 0.002 & 0.000 \\
& Media & 0.001 & 0.017 & 0.005 \\
& Baja & 0.000 & 0.003 & 0.003 \\
Baja & Alta & 0.000 & 0.002 & 0.000 \\
& Media & 0.000 & 0.006 & 0.009 \\
& Baja & 0.000 & 0.002 & 0.006 \\
\hline
\end{tabular}


Cuadro 4. Porcentaje de viviendas de cada tipo en cada uno de los municipios de Baja California Sur

(finaliza)

\begin{tabular}{|c|c|c|c|c|}
\hline \multicolumn{5}{|c|}{ Comondú } \\
\hline \multirow{2}{*}{$\begin{array}{c}\text { Tipo de } \\
\text { pared }\end{array}$} & \multirow{2}{*}{$\begin{array}{l}\text { Tipo de } \\
\text { piso }\end{array}$} & \multicolumn{3}{|c|}{ Tipo de techo } \\
\hline & & Alta & Media & Baja \\
\hline \multirow{3}{*}{ Alta } & Alta & 0.226 & 0.034 & 0.003 \\
\hline & Media & 0.273 & 0.309 & 0.003 \\
\hline & Baja & 0.005 & 0.014 & 0.002 \\
\hline \multirow{3}{*}{ Media } & Alta & 0.000 & 0.004 & 0.001 \\
\hline & Media & 0.000 & 0.059 & 0.008 \\
\hline & Baja & 0.000 & 0.018 & 0.002 \\
\hline \multirow{3}{*}{ Baja } & Alta & 0.000 & 0.001 & 0.000 \\
\hline & Media & 0.000 & 0.006 & 0.009 \\
\hline & Baja & 0.000 & 0.005 & 0.020 \\
\hline \multicolumn{5}{|c|}{ Mulegé } \\
\hline \multirow{2}{*}{$\begin{array}{c}\text { Tipo de } \\
\text { pared }\end{array}$} & \multirow{2}{*}{$\begin{array}{l}\text { Tipo de } \\
\text { piso }\end{array}$} & \multicolumn{3}{|c|}{ Tipo de techo } \\
\hline & & Alta & Media & Baja \\
\hline \multirow{3}{*}{ Alta } & Alta & 0.096 & 0.134 & 0.000 \\
\hline & Media & 0.121 & 0.344 & 0.017 \\
\hline & Baja & 0.002 & 0.012 & 0.001 \\
\hline \multirow{3}{*}{ Media } & Alta & 0.000 & 0.021 & 0.000 \\
\hline & Media & 0.002 & 0.123 & 0.021 \\
\hline & Baja & 0.000 & 0.007 & 0.011 \\
\hline \multirow{3}{*}{ Baja } & Alta & 0.000 & 0.006 & 0.000 \\
\hline & Media & 0.000 & 0.045 & 0.004 \\
\hline & Baja & 0.000 & 0.003 & 0.029 \\
\hline
\end{tabular}

\begin{tabular}{|c|c|c|c|c|}
\hline \multicolumn{5}{|c|}{ Loreto } \\
\hline \multirow{2}{*}{$\begin{array}{c}\text { Tipo de } \\
\text { pared }\end{array}$} & \multirow{2}{*}{$\begin{array}{l}\text { Tipo de } \\
\text { piso }\end{array}$} & \multicolumn{3}{|c|}{ Tipo de techo } \\
\hline & & Alta & Media & Baja \\
\hline \multirow{3}{*}{ Alta } & Alta & 0.223 & 0.080 & 0.006 \\
\hline & Media & 0.191 & 0.229 & 0.030 \\
\hline & Baja & 0.007 & 0.009 & 0.005 \\
\hline \multirow{3}{*}{ Media } & Alta & 0.000 & 0.003 & 0.000 \\
\hline & Media & 0.000 & 0.053 & 0.034 \\
\hline & Baja & 0.000 & 0.026 & 0.032 \\
\hline \multirow{3}{*}{ Baja } & Alta & 0.000 & 0.010 & 0.000 \\
\hline & Media & 0.001 & 0.008 & 0.004 \\
\hline & Baja & 0.000 & 0.003 & 0.047 \\
\hline
\end{tabular}

Con esta información y de acuerdo con las ecuaciones (4) y (5) se calculó el riesgo para cada municipio; los resultados pueden observarse en el cuadro 5.

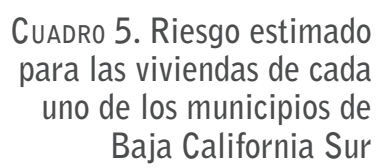

$\begin{array}{lc}\text { Municipio } & \text { Riesgo } \\ \text { Los Cabos } & 0.021 \\ \text { La Paz } & 0.023 \\ \text { Comondú } & 0.020 \\ \text { Loreto } & 0.011 \\ \text { Mulegé } & 0.013\end{array}$




\section{Discusión}

Baja California Sur es un estado con baja marginación, y aunque se encuentra altamente expuesto al impacto de ciclones tropicales, cuenta con un bajo número de viviendas precarias (paredes, pisos y techos de materiales endebles). El municipio de Loreto es el que tiene un mayor porcentaje de este tipo de viviendas (4.7\%), seguido por Mulegé (2.9\%) y muy de cerca por Los Cabos (2.8\%).

Por otro lado, el municipio de La Paz constituye el mayormente expuesto a la presencia de ciclones tropicales, pues entre 1966 y 2015 han pasado por él 24, o sea, en promedio es impactado por un ciclón cada dos años. Este municipio es el que recibe con mayor frecuencia el impacto de huracanes de categoría 1 con un periodo de retorno de 5.6 años, y a pesar de que presenta una baja proporción de viviendas construidas con materiales endebles, se sitúa en el primer lugar de riesgo en el estado.

El municipio de Los Cabos ha sido impactado por 13 ciclones en los últimos 50 años, representando el que recibe más frecuentemente huracanes de categorías 2 y 3 , con periodos de retorno de 12.5 y 25 años, respectivamente. Esto, aunado a las condiciones de sus viviendas, lo ubica en el segundo lugar de riesgo.

Por su parte, en el municipio de Comondú ha predominado el impacto de tormentas tropicales, con un periodo de retorno de 5.6 años, pero también ha sido azotado por huracanes de categorías 1 y 2 .

Dada la situación geográfica del municipio de Mulegé, aunque recibe una gran cantidad de impactos (18 en los últimos 50 años), cuando un ciclón llega, por lo general ya se ha debilitado, sea porque ha ingresado a tierra más al sur, perdiendo intensidad, o porque pasa por aguas más frías antes de golpear la península, por lo cual en este municipio ha predominado el impacto de depresiones tropicales. No obstante, el huracán Olivia (1967) tocó este municipio con categoría 3, luego de intensificarse en las cálidas aguas del Golfo de California. Aun cuando se sitúa en segundo lugar en cuanto a viviendas precarias y en número de impactos, al ser estos de baja intensidad el nivel de riesgo es menor, quedando en cuarto lugar.

El municipio de Loreto presenta el menor nivel de riesgo de la entidad, puesto que nunca ha recibido el impacto directo de huracanes de categorías 2 y 3. Siendo el municipio con mayor número de viviendas precarias en todo el estado, el impacto de huracanes de alta intensidad tendría como consecuencia pérdidas considerables. 


\section{Comentario final}

Las acciones de prevención oportuna y organizada reducen los daños en forma apreciable. Por ello es importante conocer el riesgo en que se encuentra una población para así establecer las medidas más adecuadas.

Las viviendas deben poseer características acordes con la región, a fin de disminuir los daños probables, muchas veces incrementados por el uso inapropiado de los materiales de construcción.

Para un cálculo más detallado de la vulnerabilidad es necesario realizar trabajo de campo que proporcione las características específicas de cada vivienda, así como su ubicación, con el propósito de estimar el riesgo considerando la altitud en la que se localiza, la presencia de arroyos cercanos, el tipo de suelo y la geomorfología de la zona como factores de riesgo. Asimismo, es menester efectuar estudios que permitan determinar la vulnerabilidad social y económica de la población.

\section{Fuentes consultadas}

Cenapred. (2009). Características e impacto socioeconómico de los principales desastre ocurridos en la república mexicana en el año 2007. México: Centro Nacional de Prevención de Desastres. Recuperado de http:// www.proteccioncivil.gob.mx/work/models/ProteccionCivil/Resource/375/1/images/no_9.pdf

Cenapred. (s. f.). Seguridad en las Construcciones. En Centro Nacional de Prevención de Desastres. Recuperado de http://www.cenapred.unam.mx/ es/PreguntasFrecuentes/faqpopo3.html [2016, 11 de enero].

Chardon, A.-C. y González, J. L. (2002). Programa de Información e Indicadores de Gestión de Riesgos. Indicadores para la Gestión de Riesgos. Amena$z a$, vulnerabilidad, riesgo, desastre, mitigación, prevención.... Primer acercamiento a conceptos, características y metodologías de análisis y evaluación. Manizales: Banco Interamericano de Desarrollo/Universidad Nacional de Colombia-Sede Manizales/Instituto de Estudios Ambientales. Recuperado de http://idea.unalmzl.edu.co/documentos/ Anne-Catherine\%20fase\%20I.pdf_[2016, 15 de abril]. 
CNNMéxico. (22 de septiembre de 2014). Dos extranjeros más mueren por el paso de “Odile” en Baja California Sur. Recuperado de http://mexico. cnn.com/nacional/2014/09/22/zona-de-desastre-en-municipios-debaja-california-sur-por-odile [2015, 25 de julio].

Flores Corona, L. et al. (2004). Guía básica para la elaboración de atlas estatales y municipales de peligros y riesgos. México: Centro Nacional de Prevención de Desastres. Recuperado de http://www.cenapred.unam.mx/es/ DocumentosPublicos/PDF/SerieEspecial/metodologiasAtlas.pdf [2015, 16 de agosto].

InEgi. (2010). Censo de Población y Vivienda 2010. México: Instituto Nacional de Estadística y Geografía.

Mendoza-Estrada, D. R., Vélez-Morales, L., Fuentes-Mariles, O. A., De LunaCruz, F. y Pelaez-Pavón, L. B. (2014). Modelo para la estimación de escenarios temporales del riesgo por viento de ciclones tropicales. En XXIII Congreso Nacional de Hidráulica, México.

oea. (1993). Manual Sobre el Manejo de Peligros Naturales en la Planificación para el Desarrollo Regional Integrado. Washington, D. C.: Departamento de Desarrollo Regional y Medio Ambiente-Secretaría Ejecutiva para Asuntos Económicos y Sociales-Organización de los Estados Americanos/Oficina de Asistencia para Desastres en el Extranjero-Agencia de los Estados Unidos para el Desarrollo Internacional. Recuperado de http://www.oas.org/dsd/publications/unit/oea65s/oea65s.pdf [2015, 5 de agosto].

Rodríguez, G. (11 de octubre de 2008). Azota Norbert a BCs en Comondú y Loreto. El Universal. Recuperado de http://archivo.eluniversal.com. $\mathrm{mx} /$ notas/546086.html_2015, 19 de julio].

Romero, G. y Maskrey, A. (1993). Como entender los desastres naturales. En A. Maskrey (comp.), Los desastres no son naturales (pp. 6-10). Red de Estudios Sociales en Prevención de Desastres en América Latina. Recuperado de http://www.oei.es/decada/portadas/Desnat.pdf [2016, 15 de abril].

Romero-Vadillo, E. (2015). Los ciclones tropicales en el Pacífico mexicano. Su impacto en Baja California Sur. México. México: Universidad Autónoma de Baja California Sur. 
Romero-Vadillo, I. y Romero-Vadillo, E. (2014). Fenómenos hidrometerológicos como factor de incremento de la marginación. En Memorias en extenso del $2^{\circ}$ Congreso Internacional de la Red del Medio Ambiente. Instituto Politécnico Nacional, México.

Velasco, B. (6 de octubre de 2014). Daños a Bcs por Odile ascienden a 7 mil 863 mdp: Fonden. El Financiero. Recuperado de http://www.elfinanciero. com.mx/sociedad/danos-a-bcs-por-odile-ascienden-a-mil-863-millones-de-pesos.html [2015, 25 de julio].

Wisner, B., Blaikie, P., Cannon, T. y Davis, I. (2003). At Risk: Natural hazards, people's vulnerability and disasters ( $2^{\text {nd }}$ ed.). Retrieved from http:// www.preventionweb.net/files/670_72351.pdf [2016, April 15 $\left.{ }^{\text {th }}\right]$. 